\title{
Modular Adjacency Algebras of Hamming Schemes
}

\author{
MASAYOSHI YOSHIKAWA \\ yoshi@math.shinshu-u.ac.jp \\ Department of Mathematical Sciences, Faculty of Science, Shinshu University, Matsumoto 390-8621, Japan
}

Received January 15, 2003; Revised October 27, 2003; Accepted October 27, 2003

\begin{abstract}
To each association scheme $G$ and to each field $R$, there is associated naturally an associative algebra, the so-called adjacency algebra $R G$ of $G$ over $R$. It is well-known that $R G$ is semisimple if $R$ has characteristic 0 . However, little is known if $R$ has positive characteristic. In the present paper, we focus on this case. We describe the algebra $R G$ if $G$ is a Hamming scheme (and $R$ a field of positive characteristic). In particular, we show that, in this case, $R G$ is a factor algebra of a polynomial ring by a monomial ideal.
\end{abstract}

Keywords: association scheme, Hamming scheme, modular adjacency algebra

\section{Introduction}

Let $p$ be a prime number, and let $\mathbb{F}_{p}$ denote a field with $p$ elements. Let $n$ and $q$ be positive integers, and let $H(n, q)$ denote the Hamming scheme the point set of which consists of all $n$-tuples of elements of $\{0,1, \ldots, q-1\}$. It follows from [2, III.Theorem 2.3] that the Frame number of $H(n, q)$ is $q^{n(n+1)}$. Therefore from [1, Theorem 1.1] or [5, Theorem 4.2], we know that $\mathbb{F}_{p} H(n, q)$ is semisimple iff $p$ does not divide $q$. Moreover, in Section 2.3 of the present paper, we shall show that, if $p$ divides $q, \mathbb{F}_{p} H(n, p) \cong \mathbb{F}_{p} H(n, q)$. Therefore, we shall focus our attention to the investigation of $\mathbb{F}_{p} H(n, p)$.

From [4, Theorem 3.4, Corollary 3.5] we know that $\mathbb{F}_{p}$ is a splitting field for $\mathbb{F}_{p} H(n, p)$. Therefore, if we determine the structure of $\mathbb{F}_{p} H(n, p)$, we know the structure over any field of characteristic $p$.

We will describe $\mathbb{F}_{p} H(n, p)$ as a factor algebra of a polynomial ring by a monomial ideal for the clarity of the structure. A monomial ideal is the ideal that is generated by only monomials.

\section{Preparation}

For the definitions in this section, refer to [2].

\subsection{Association schemes}

Let $\mathrm{X}$ be a finite set of cardinality $n$. We define $R_{0}:=\{(x, x) \mid x \in X\}$. Let $R_{i} \subseteq X \times X$ be given. We set $R_{i}^{*}:=\left\{(z, y) \mid(y, z) \in R_{i}\right\}$. Let $G$ be a partition of $X \times X$ such that $R_{0} \in G$ and the empty set $\emptyset \notin G$, and assume that, $R_{i}^{*} \in G$ for each $R_{i} \in G$. Then, the pair $(X, G)$ 
will be called an association scheme if, for all $R_{i}, R_{j}, R_{k} \in G$, there exists an integer $p_{i j k}$ such that, for all $y, z \in X$

$$
(y, z) \in R_{k} \Rightarrow \sharp\left\{x \in X \mid(y, x) \in R_{i},(x, z) \in R_{j}\right\}=p_{i j k} .
$$

The elements of $\left\{p_{i j k}\right\}$ will be called the intersection numbers of $(X, G)$.

For each $R_{i} \in G$, we define the $n \times n$ matrix $A_{i}$ indexed by the elements of $X$,

$$
\left(A_{i}\right)_{x y}= \begin{cases}1 & \text { if }(x, y) \in R_{i} \\ 0 & \text { otherwise }\end{cases}
$$

and this matrix $A_{i}$ will be called the adjacency matrix of $R_{i}$

Let the cardinal number of $G$ be $d+1$ and let $\mathrm{J}$ be the $n \times n$ all 1 matrix. Then, by the definition, it follows that $\sum_{i=0}^{d} A_{i}=J$. It follows that for all $A_{i}, A_{j}$,

$$
A_{i} A_{j}=\sum_{k=0}^{d} p_{i j k} A_{k}
$$

From this fact, we can define an algebra naturally. For the commutative ring $R$ with 1 , we put $R(X, G)=\bigoplus_{i=0}^{d} R A_{i}$ as a matrix ring over $R$, and it will be called the adjacency algebra of $(X, G)$ over $R$.

For all $i, j, k \in\{0,1, \ldots, d\}$, we define the matrix $B_{i}$ by $\left(B_{i}\right)_{j k}=p_{i j k}$. This matrix $B_{i}$ will be called the $i$-th intersection matrix. It follows that for all $B_{i}, B_{j}, B_{i} B_{j}=\sum_{k=0}^{d} p_{i j k} B_{k}$. Therefore we can define an algebra $R B=\bigoplus_{i=0}^{d} R B_{i}$ for a commutative ring $R$ with 1 , and it will be called the intersection algebra of $(X, G)$ over $R$. Then the mapping from the adjacency algebra to the intersection algebra of $(X, G)$ over $R, A_{i} \mapsto B_{i}$, is an algebra isomorphism.

\subsection{P-polynomial schemes}

A symmetric association scheme $\left(X,\left\{R_{i}\right\}_{0 \leq i \leq d}\right)$ is called a $P$-polynomial scheme with respect to the ordering $R_{0}, R_{1}, \ldots, R_{d}$, if there exist some complex coefficient polynomials $v_{i}$ of degree $i(0 \leq i \leq d)$ such that $A_{i}=v_{i}\left(A_{1}\right)$, where $A_{i}$ is the adjacency matrix of $R_{i}$.

We use the following notation: a tridiagonal matrix

$$
B=\left(\begin{array}{ccccc}
a_{0} & c_{1} & & & 0 \\
b_{0} & a_{1} & \ddots & & \\
& b_{1} & \ddots & \ddots & \\
& & \ddots & \ddots & c_{d} \\
0 & & & b_{d-1} & a_{d}
\end{array}\right)
$$


is denoted by

$$
\left\{\begin{array}{ccccc}
* & c_{1} & \cdots & c_{d-1} & c_{d} \\
a_{0} & a_{1} & \cdots & a_{d-1} & a_{d} \\
b_{0} & b_{1} & \cdots & b_{d-1} & *
\end{array}\right\} .
$$

Then the following (i) and (ii) are equivalent to each other (see [2, Proposition 1.1]). (i) $B_{1}$ is a tridiagonal matrix with non-zero off-diagonal entries:

$$
\left\{\begin{array}{cccccc}
* & 1 & c_{2} & \cdots & c_{d-1} & c_{d} \\
0 & a_{1} & a_{2} & \cdots & a_{d-1} & a_{d} \\
b_{0} & b_{1} & b_{2} & \cdots & b_{d-1} & *
\end{array}\right\}\left(b_{i} \neq 0, c_{i} \neq 0\right)
$$

(ii) $\left(X,\left\{R_{i}\right\}_{0 \leq i \leq d}\right)$ is a $P$-polynomial scheme with respect to the ordering $R_{0}, R_{1}, \ldots, R_{d}$, i.e.,

$$
A_{i}=v_{i}\left(A_{1}\right) \quad(i=0,1, \ldots, d)
$$

for some polynomials $v_{i}$ of degree $i$.

\subsection{Hamming schemes}

Let $\Sigma$ be an alphabet of $q$ symbols $\{0,1, \ldots, q-1\}$. We define $\Omega$ to be the set $\Sigma^{n}$ of all $n$-tuples of elements of $\Sigma$, and let $\rho(x, y)$ be the number of coordinate places in which the $n$-tuples $x$ and $y$ differ. Thus $\rho(x, y)$ is the Hamming distance between $x$ and $y$. we set

$$
R_{i}=\{(x, y) \in \Omega \times \Omega \mid \rho(x, y)=i\},
$$

and then $\left(\Omega,\left\{R_{i}\right\}_{0 \leq i \leq n}\right)$ is an association scheme. This will be called the Hamming scheme, and denoted by $H(n, q)$.

We consider the intersection numbers $p_{i j k}^{(n, q)}$ of $H(n, q)$. For the convenience of the argument, we extend the binomial coefficient as follows.

$$
\left(\begin{array}{l}
0 \\
x
\end{array}\right)= \begin{cases}1 & \text { if } x=0 \\
0 & \text { otherwise }\end{cases}
$$

and for each integer $x$ and each negative integer $y$,

$$
\left(\begin{array}{l}
x \\
y
\end{array}\right)=0, \quad\left(\begin{array}{l}
y \\
x
\end{array}\right)=0
$$


We consider the following three elements in $\Omega$,

$$
\begin{aligned}
& (\overbrace{0,0, \ldots, 0}^{k}, 0, \ldots, 0), \\
& \left(\alpha_{0}, \alpha_{1}, \alpha_{*}, \beta\right), \\
& (\underbrace{1,1, \ldots, 1}_{k}, 0, \ldots, 0),
\end{aligned}
$$

where $\alpha_{0}, \alpha_{1}, \alpha_{*}$, and $\beta$ means that there are $\alpha_{0} 0$ 's, $\alpha_{1} 1$ 's, and $\alpha_{*}$ other symbols among first $k$ figures, and $\beta$ non-zero symbols among remaining $(n-k)$ figures.

Then if we assume that

$$
\begin{aligned}
& \left((0, \ldots, 0),\left(\alpha_{0}, \alpha_{1}, \alpha_{*}, \beta\right)\right) \in R_{i} \\
& \left(\left(\alpha_{0}, \alpha_{1}, \alpha_{*}, \beta\right),(1, \ldots, 1,0, \ldots, 0)\right) \in R_{j} \\
& ((0, \ldots, 0),(1, \ldots, 1,0, \ldots, 0)) \in R_{k}
\end{aligned}
$$

the system of equations must hold that

$$
\left\{\begin{array}{l}
\alpha_{1}+\alpha_{*}+\beta=i \\
\alpha_{0}+\alpha_{*}+\beta=j \\
\alpha_{0}+\alpha_{1}+\alpha_{*}=k
\end{array}\right.
$$

From the definition, since $p_{i j k}^{(n, q)}$ is the total of $n$-tuples that satisfy the above the system of equations,

$$
p_{i j k}^{(n, q)}=\sum_{\beta=0}^{n-k}\left(\begin{array}{c}
k \\
k-i+\beta
\end{array}\right)\left(\begin{array}{c}
i-\beta \\
k-j+\beta
\end{array}\right)\left(\begin{array}{c}
n-k \\
\beta
\end{array}\right)(q-1)^{\beta}(q-2)^{i+j-k-2 \beta}
$$

Therefore if $p \mid q$ for some prime number $p, p_{i j k}^{(n, q)} \equiv p_{i j k}^{(n, p)} \quad(\bmod p)$. Since the intersection numbers are the structure constants of the adjacency algebra, $\mathbb{F}_{p} H(n, q) \cong \mathbb{F}_{p} H(n, p)$.

The Hamming scheme $H(n, q)$ is a $P$-polynomial scheme (see [2]), and

$$
B_{1}=\left\{\begin{array}{cccccc}
* & 1 & \cdots & i & \cdots & n \\
0 & q-2 & \cdots & i(q-2) & \cdots & n(q-2) \\
n(q-1) & (n-1)(q-1) & \cdots & (n-i)(q-1) & \cdots & *
\end{array}\right\} .
$$

For the remainder of this paper, let $p$ be a fixed prime number. Therefore we set $H(n):=H(n, p)$. And we denote the intersection numbers, the adjacency matrices, and the intersection matrices of $H(n)$ respectively by $p_{i j k}^{(n)}, A_{i}^{(n)}, B_{i}^{(n)}$ and so on. 
If we index the adjacency matrices by a suitable order, for example, the lexicographic order on $\Sigma^{n}$, then it follows that

$$
A_{i}^{(n+1)}=I \otimes A_{i}^{(n)}+K \otimes A_{i-1}^{(n)} \quad \text { for } \forall i \in\{0,1, \ldots, n+1\},
$$

where $I$ is the $p \times p$ identity matrix, $K$ is the $p \times p$ matrix such that the diagonal entries are 0 and the others $1, A_{-1}^{(n)}=A_{n+1}^{(n)}=O$ (the $p^{n} \times p^{n}$ zero matrix), and $\otimes$ is the Kronecker product. The Kronecker product $A \otimes B$ of matrices $A$ and $B$ is defined as follows. Suppose $A=\left(a_{i j}\right)$. Then $A \otimes B$ is obtained by replacing the entry $a_{i j}$ of $A$ by the matrix $a_{i j} B$, for all $i$ and $j$. The most important property of this product is that, provided the required products exist,

$(A \otimes B)(X \otimes Y)=A X \otimes B Y$.

\section{3. $H\left(p^{r}-1\right)$}

The intersection numbers are the structure constants of the adjacency algebra. Therefore, if we consider the adjacency algebra over a field of characteristic $p$, we may consider the intersection numbers modulo $p$.

The size of the adjacency matrix of $H(n)$ is $p^{n}$. Therefore, the adjacency algebra of $H(n)$ over a field of characteristic $p$ is local. Moreover the unique irreducible representation is $A_{i} \mapsto p_{i i^{*} 0}$ (see [4, Theorem 3.4, Corollary 3.5]). Therefore $\mathbb{F}_{p}$ is a splitting field for $\mathbb{F}_{p} H(n)$. Thus, if we determine the structure of $\mathbb{F}_{p} H(n)$, we know the structure over any field of characteristic $p$.

For the remainder of this paper, since we consider the adjacency algebras only over $\mathbb{F}_{p}$, we set $\mathfrak{A}_{n}:=\mathbb{F}_{p} H(n)$.

By the definition,

$$
B_{1}^{\left(p^{r}-1\right)}=\left(\begin{array}{llll}
B_{1}^{(p-1)} & & & \\
& B_{1}^{(p-1)} & & \\
& & \ddots & \\
& & & B_{1}^{(p-1)}
\end{array}\right),
$$

therefore if we set $A_{i}^{(p-1)}=v_{i}\left(A_{1}^{(p-1)}\right)$, it follows that for $0 \leq \alpha \leq p-1$,

$$
A_{p i+\alpha}^{\left(p^{r}-1\right)}=v_{\alpha}\left(A_{1}^{\left(p^{r}-1\right)}\right) A_{p i}^{\left(p^{r}-1\right)}
$$

Then since any $c_{i}^{(p-1)} \not \equiv 0 \quad(\bmod p)$, we can define $v_{\alpha}$ over $\mathbb{F}_{p}$ for $0 \leq \alpha \leq p-1$. For calculating $B_{p i+\alpha}^{\left(p^{r}-1\right)}$, we prepare the following theorem and corollary. 
Theorem 1 (Lucas' theorem [3, Theorem 3.4.1]) Let $p$ be prime, and let

$$
\begin{aligned}
m & =a_{0}+a_{1} p+\cdots+a_{k} p^{k}, \\
n & =b_{0}+b_{1} p+\cdots+b_{k} p^{k},
\end{aligned}
$$

where $0 \leq a_{i}, b_{i}<p$ for $i=0,1, \ldots, k-1$. Then

$$
\left(\begin{array}{l}
m \\
n
\end{array}\right) \equiv \prod_{i=0}^{k}\left(\begin{array}{l}
a_{i} \\
b_{i}
\end{array}\right)(\bmod \mathrm{p}) .
$$

Corollary 2 Let $p, m$, and $n$ be as in Theorem 1. Then, for any two elements $\alpha$ and $\beta$ in $\{0,1, \ldots, p-1\}$, we have

$$
\left(\begin{array}{c}
p m+\alpha \\
p n+\beta
\end{array}\right) \equiv\left(\begin{array}{l}
m \\
n
\end{array}\right)\left(\begin{array}{c}
\alpha \\
\beta
\end{array}\right)(\bmod \mathrm{p})
$$

Now we want to culculate $B_{p i+\alpha}^{\left(p^{r}-1\right)}$, that is the coefficients of $A_{p i+\alpha}^{\left(p^{r}-1\right)} A_{p j+\beta}^{\left(p^{r}-1\right)}$. But it is enough to investigate $A_{p i}^{\left(p^{r}-1\right)} A_{p j}^{\left(p^{r}-1\right)}$, i.e. $p_{p i p j k}^{\left(p^{r}-1\right)}$ because we know $v_{\alpha}\left(A_{1}^{\left(p^{r}-1\right)}\right) v_{\beta}\left(A_{1}^{\left(p^{r}-1\right)}\right)$.

Here we recall from Section 2.3 that

$$
\begin{aligned}
p_{p i p j k}^{\left(p^{r}-1\right)}= & p_{p i p j k}^{\left(p^{r}-1, p\right)}=\sum_{s=0}^{p^{r}-1-k}\left(\begin{array}{c}
k \\
k-p i+s
\end{array}\right)\left(\begin{array}{c}
p i-s \\
k-p j+s
\end{array}\right)\left(\begin{array}{c}
p^{r}-1-k \\
s
\end{array}\right) \\
& \times(p-1)^{s}(p-2)^{p i+p j-k-2 s} .
\end{aligned}
$$

We assume that $k=k^{\prime}+p k^{\prime \prime}$ and $s=s^{\prime}+p s^{\prime \prime}$ where $0 \leq k^{\prime}, s^{\prime}<p$. Then by Corollary 2 , it follows that

$$
\begin{array}{r}
0<s^{\prime}<p-k^{\prime} \Rightarrow\left(\begin{array}{c}
k \\
k-p i+s
\end{array}\right) \equiv 0 \quad(\bmod p), \\
p-1-k^{\prime}<s^{\prime}<p \Rightarrow\left(\begin{array}{c}
p^{r}-1-k \\
s
\end{array}\right) \equiv 0 \quad(\bmod p),
\end{array}
$$

and if $s^{\prime}=0$,

$$
k^{\prime} \neq 0 \Rightarrow\left(\begin{array}{c}
p i-s \\
k-p j+s
\end{array}\right) \equiv 0 \quad(\bmod p)
$$


Therefore it follows that if $k=p k^{\prime \prime}$,

$$
\begin{aligned}
p_{p i p j k}^{\left(p^{r}-1\right)}= & \sum_{s=0}^{p^{r}-1-k}\left(\begin{array}{c}
k \\
k-p i+s
\end{array}\right)\left(\begin{array}{c}
p i-s \\
k-p j+s
\end{array}\right)\left(\begin{array}{c}
p^{r}-1-k \\
s
\end{array}\right) \\
& \times(p-1)^{s}(p-2)^{p i+p j-k-2 s} \\
\equiv & \sum_{s^{\prime \prime}=0}^{p^{r-1}-1-k^{\prime \prime}}\left(\begin{array}{c}
p k^{\prime \prime} \\
p k^{\prime \prime}-p i+p s^{\prime \prime}
\end{array}\right)\left(\begin{array}{c}
p i-p s^{\prime \prime} \\
p k^{\prime \prime}-p j+p s^{\prime \prime}
\end{array}\right)\left(\begin{array}{c}
p^{r}-1-p k^{\prime \prime} \\
p s^{\prime \prime}
\end{array}\right) \\
& \times(p-1)^{p s^{\prime \prime}}(p-2)^{p i+p j-p k^{\prime \prime}-2 p s^{\prime \prime}} \\
\equiv & \sum_{s^{\prime \prime}=0}^{p^{r-1}-1-k^{\prime \prime}}\left(\begin{array}{c}
k^{\prime \prime} \\
k^{\prime \prime}-i+s^{\prime \prime}
\end{array}\right)\left(\begin{array}{c}
i-s^{\prime \prime} \\
k^{\prime \prime}-j+s^{\prime \prime}
\end{array}\right)\left(\begin{array}{c}
p^{r-1}-1-k^{\prime \prime} \\
s^{\prime \prime}
\end{array}\right)\left(\begin{array}{c}
p-1 \\
0
\end{array}\right) \\
& \times(p-1)^{s^{\prime \prime}}(p-2)^{i+j-k^{\prime \prime}-2 s^{\prime \prime}} \\
\equiv & p_{i j k^{\prime \prime}}^{\left(p^{r-1}-1\right)}(\bmod p),
\end{aligned}
$$

and if $p \nmid k, p_{p i}^{\left(p^{r}-1\right)} \equiv 0 \quad(\bmod p)$.

Thus

$$
\begin{aligned}
A_{p i+\alpha}^{\left(p^{r}-1\right)} A_{p j+\beta}^{\left(p^{r}-1\right)} & =v_{\alpha}\left(A_{1}^{\left(p^{r}-1\right)}\right) v_{\beta}\left(A_{1}^{\left(p^{r}-1\right)}\right) A_{p i}^{\left(p^{r}-1\right)} A_{p j}^{\left(p^{r}-1\right)} \\
& \equiv \sum_{k=0}^{p^{r-1}-1} \sum_{\gamma=0}^{p-1} p_{i j k}^{\left(p^{r-1}-1\right)} p_{\alpha \beta \gamma}^{(p-1)} A_{p k+\gamma}^{\left(p^{r}-1\right)} .
\end{aligned}
$$

By the above argument, it follows that

$$
B_{p i+\alpha}^{\left(p^{r}-1\right)}=B_{i}^{\left(p^{r-1}-1\right)} \otimes B_{\alpha}^{(p-1)} .
$$

Repeating the same argument, we know that for each non-negative integer $m$ such that $0 \leq m \leq p^{r}-1$ and $m=m_{0} p^{0}+m_{1} p^{1}+\cdots+m_{r-1} p^{r-1}$,

$$
B_{m}^{\left(p^{r}-1\right)}=B_{m_{r-1}}^{(p-1)} \otimes B_{m_{r-2}}^{(p-1)} \otimes \cdots \otimes B_{m_{0}}^{(p-1)} .
$$

From this fact, we obtain that

$$
\mathfrak{A}_{p^{r}-1} \cong \overbrace{\mathfrak{A}_{p-1} \otimes \mathfrak{A}_{p-1} \otimes \cdots \otimes \mathfrak{A}_{p-1}}^{r} .
$$

Theorem $3 \quad \mathfrak{A}_{p-1} \cong \mathbb{F}_{p} C_{p} \cong \mathbb{F}_{p}[X] /\left\langle X^{p}\right\rangle$

Proof: Since $B_{1}^{(p-1)}-B_{0}^{(p-1)}$ is nilpotent and its rank is $p-1$, the theorem holds. 
Therefore the following theorem holds.

Theorem 4 For each positive integer $r, \mathfrak{A}_{p^{r-1}}$ is isomorphic to the group algebra of the elementary abelian group of order $p^{r}$ over $\mathbb{F}_{p}$.

\section{The structure of $\mathfrak{A}_{n}$}

In the previous section, we considered the structure of $\mathfrak{A}_{p^{r}-1}$. To determine the structure of $\mathfrak{A}_{n}$, in general, we construct an algebra homomorphism $\mathfrak{A}_{n+1} \rightarrow \mathfrak{A}_{n}$.

From Section 2.3, $A_{i}^{(n+1)}=I \otimes A_{i}^{(n)}+K \otimes A_{i-1}^{(n)}$. This means that $\mathfrak{A}_{n+1}$ is a subalgebra of $\mathfrak{A}_{1} \otimes \mathfrak{A}_{n}$. The unique irreducible representation of $\mathfrak{A}_{1}$ is $A_{0}^{(1)} \mapsto 1, A_{1}^{(1)} \mapsto-1$.

Therefore we can define naturally the mapping $f_{n+1}$ for each positive integer $n$ by

$$
\begin{aligned}
& f_{n+1}: \mathfrak{A}_{n+1} \rightarrow \mathfrak{A}_{n} \\
& A_{i}^{(n+1)}=I \otimes A_{i}^{(n)}+K \otimes A_{i-1}^{(n)} \mapsto A_{i}^{(n)}-A_{i-1}^{(n)} .
\end{aligned}
$$

Proposition 5 For each positive integer $n, f_{n+1}: \mathfrak{A}_{n+1} \rightarrow \mathfrak{A}_{n}$ above is an algebra epimorphism.

By Theorem 4, $\mathfrak{A}_{p^{r}-1}$ is isomorphic to $\mathbb{F}_{p}(\underbrace{}_{c_{p} \times C_{p} \times \cdots \times C_{p}})$ for each positive integer $r$. Let $x_{1}, x_{2}, \ldots, x_{r}$ be the generators of each $C_{p}$ starting from the right. Then the element of $\mathfrak{A}_{p^{r}-1}$ corresponding to $x_{i}$ by the algebra homomorphism above, is $A_{p^{i-1}}^{\left(p^{r}-1\right)}$.

From the representation theory of the finite group, there exists the algebra isomorphism $g$ from the quotient ring $\mathfrak{P}_{r}=F_{p}\left[X_{1}, X_{2}, \ldots, X_{r}\right] /\left\langle X_{1}^{p}, \cdots, X_{r}^{p}\right\rangle$ of the polynomial ring of $r$ variables over $\mathbb{F}_{p}$ to $\mathbb{F}_{p}(\underbrace{c_{p \times c_{p} \times \cdots \times c_{p}}})$ by $g\left(X_{i}\right)=1-x_{i}$. Therefore we can define an algebra isomorphism $s_{r}: \mathfrak{P}_{r} \rightarrow{\mathfrak{\mathfrak { A } ^ { r }}}_{p^{r}-1}$ by

$$
s_{r}\left(X_{i}\right)=A_{0}^{\left(p^{r}-1\right)}-A_{p^{i-1}}^{\left(p^{r}-1\right)}
$$

We define a weight function $w t$ on the set of the monomials of $\mathfrak{P}_{r}$ by

$$
w t\left(X_{i}\right)=p^{i-1}, \quad w t\left(\prod_{j} X_{j}^{k_{j}}\right)=\sum_{j} k_{j} p^{j-1} .
$$

Proposition 6 For each positive integer $m$ such that $1 \leq m \leq p-1$,

$$
\left(A_{0}^{\left(p^{r}-1\right)}-A_{p^{i}}^{\left(p^{r}-1\right)}\right)^{m}=m ! \sum_{n=0}^{m}\left(\begin{array}{c}
m \\
n
\end{array}\right)(-1)^{n} A_{n p^{i}}^{\left(p^{r}-1\right)} .
$$


And if $i \neq j, 0 \leq \alpha, \beta \leq p-1$,

$$
A_{\alpha p^{i}}^{\left(p^{r}-1\right)} A_{\beta p^{j}}^{\left(p^{r}-1\right)}=A_{\alpha p^{i}+\beta p^{j}}^{\left(p^{r}-1\right)}
$$

Proof: We obtain the first equation by the induction and the second equation by considering tensor expression of $B_{\alpha p^{i}}^{\left(p^{r}-1\right)}$ (see Section 3).

Let $Y_{i}=X_{i_{0}}^{k_{0}} X_{i_{1}}^{k_{1}} \ldots X_{i_{s}}^{k_{s}}$ be the monomial of $\mathfrak{P}_{r}$ such that $w t\left(Y_{i}\right)=i$. Then by the above two equations, the following Proposition holds.

\section{Proposition 7}

$$
\begin{aligned}
s_{r}\left(Y_{i}\right) & =\prod_{j=0}^{s}\left(A_{0}^{\left(p^{r}-1\right)}-A_{p^{i_{j}-1}}^{\left(p^{r}-0\right)}\right)^{k_{j}} \\
& =\left(\prod_{j=0}^{s} k_{j} !\right) \sum_{n=0}^{p^{r}-1}\left(\begin{array}{l}
i \\
n
\end{array}\right)(-1)^{n} A_{n}^{\left(p^{r}-1\right)} .
\end{aligned}
$$

Proof: The first equation means that the expansion of $\left(A_{0}^{\left(p^{r}-1\right)}-A_{p^{i}}^{\left(p^{r}-1\right)}\right)^{m}$ is the formula that one expands $\left(X^{0}-X^{p^{i}}\right)^{m}$ and replaces $X^{n}$ with $A_{n}^{\left(p^{r}-1\right)}$ and multiplies it by $m !$. The second equation means that we can apply the same way to $\prod_{j=0}^{s}\left(A_{0}^{\left(p^{r}-1\right)}-A_{p^{i_{j}}-1}^{\left(p^{r}-1\right)}\right)^{k_{j}}$. Namely, $\prod_{j=0}^{s}\left(A_{0}^{\left(p^{r}-1\right)}-A_{p^{i_{j}}-1}^{\left(p^{r}-1\right)}\right)^{k_{j}}$ is the formula that one expands $\prod_{j=0}^{s}\left(X^{0}-X^{p^{i_{j}-1}}\right)^{k_{j}}=$ $\left(X^{0}-X^{1}\right)^{i}$ and replaces $X^{n}$ with $A_{n}^{\left(p^{r}-1\right)}$ and multiplies it by $\prod_{j=0}^{s} k_{j}$ !.

Then the following theorem, that is the main theorem in this paper, holds.

Theorem 8 We set $\mathfrak{P}=\mathbb{F}_{p}\left[X_{1}, X_{2}, \ldots\right] /\left\langle X_{1}^{p}, X_{2}^{p} \ldots\right\rangle$, and for each positive integer $n$, we set

$$
\left.W_{n}=\langle x| x \text { is the monomial of } \mathfrak{P} \text { such that } w t(x)>n\right\rangle .
$$

Then it holds that $\mathfrak{P} / W_{n} \cong \mathfrak{A}_{n}$ as algebras.

Proof: It is enough that we show that,

$\mathfrak{P}_{r} / W_{n} \cong \mathfrak{A}_{n} \quad$ for $n<p^{r}$ 
Furthermore it is enough that we show that for each positive integer $n$ such that $n \leq p^{r}-1$, $Y_{n} \in \operatorname{Ker} f_{n} f_{n+1} \ldots f_{p^{r}-1} s_{r}$. Since

$$
\begin{aligned}
f_{n} & f_{n+1} \ldots f_{p^{r}-1} s_{r}\left(Y_{n}\right) \\
& =\left(\prod_{j=0}^{s} k_{j} !\right) f_{n} f_{n+1} \ldots f_{p^{r}-1}\left(\sum_{i=0}^{p^{r}-1}\left(\begin{array}{c}
n \\
i
\end{array}\right)(-1)^{i} A_{i}^{\left(p^{r}-1\right)}\right) \\
& \left.=\left(\prod_{j=0}^{s} k_{j} !\right) f_{n} f_{n+1} \ldots f_{p^{r}-2}\left(\sum_{i=0}^{p^{r}-2}\left(\begin{array}{c}
n \\
i
\end{array}\right)(-1)^{i}-\left(\begin{array}{c}
n \\
i+1
\end{array}\right)(-1)^{i+1}\right) A_{i}^{\left(p^{r}-2\right)}\right) \\
& =\left(\prod_{j=0}^{s} k_{j} !\right)(-1) f_{n} f_{n+1} \ldots f_{p^{r}-2}\left(\sum_{i=0}^{p^{r}-2}\left(\begin{array}{c}
n+1 \\
i+1
\end{array}\right)(-1)^{i+1} A_{i}^{\left(p^{r}-2\right)}\right) \\
& =\left(\prod_{j=0}^{s} k_{j} !\right)(-1)^{p^{r}-n} \sum_{i=0}^{n-1}\left(\begin{array}{c}
p^{r} \\
i+p^{r}-n
\end{array}\right)(-1)^{i+p^{r}-n} A_{i}^{(n-1)} \\
& =0,
\end{aligned}
$$

the theorem holds.

Remark 1 We set $G_{n, q}=S_{q} w r S_{n}, H_{n, q}=S_{q-1} w r S_{n}$ for positive integers $n, q$. Let $K$ be a field. Then $K H(n, q)$ and the Hecke algebra $\operatorname{End}_{K G_{n, q}}\left(1_{H_{n, q}}^{G_{n, q}}\right)$ are isomorphic as algebras (see [2, III.2]). Therefore we also could determine the structure of $\operatorname{End}_{K G_{n, q}}\left(1_{H_{n, q}}^{G_{n, q}}\right)$. In particular, Theorem 4 means that for each positive integer $r$, if $n=p^{r}-1$, the Hecke algebra $\operatorname{End}_{\mathbb{F}_{p} G_{n, p}}\left(1_{H_{n, p}}^{G_{n, p}}\right)$ is isomorphic to the group algebra of the elementary abelian group of order $p^{r}$.

\section{Acknowledgment}

The author is thankful to Akihide Hanaki for valuable suggestions and comments and to the referee for lots of helpful remarks and suggestions.

\section{References}

1. Z. Arad, E. Fisman, and M. Muzychuk, "Generalized table algebras," Israel J. Math. 144 (1999), 29-60.

2. E. Bannai and T. Ito, Algebraic Combinatorics. I. Association Schemes, Benjamin-Cummings, Menlo Park, CA, 1984.

3. P.-J. Cameron, Combinatorics: Topics, Techniques, Algorithms, Cambridge University Press, 1994.

4. A. Hanaki, "Locality of a modular adjacency algebra of an association scheme of prime power order," Arch. Math (to appear).

5. A. Hanaki, "Semisimplicity of adjacency algebras of association schemes," J. Alg. 225 (2000), 124-129.

6. P.-H. Zieschang, An Algebraic Approach to Association Schemes, Lecture Notes in Math. vol. 1628, Springer, Berlin-Heidelberg-New York, 1996. 